\title{
A comparative study of metamodeling methods for the design optimization of variable stiffness composites
}

\author{
Mahdi Arian Nik, Kazem Fayazbakhsh, Damiano Pasini*, Larry Lessard \\ Department of Mechanical Engineering, McGill University, Macdonald Engineering Building, 817 Sherbrooke \\ West, Montreal, QC, Canada H3A 2 K6 \\ *Corresponding author: damiano.pasini@mcgill.ca; Tel: (+1) 514-398-6295; fax: (+1) 514-398-7365
}

\begin{abstract}
Automated fiber placement is a manufacturing technology that enables to build composite laminates with curvilinear fibers. To determine their optimum mechanical properties, finite element analysis is commonly used as a solver within an optimization framework. The analysis of laminates with curvilinear fibers coupled with the fiber path optimization requires a large number of function evaluations, each time-consuming. To reduce the time for analysis and thus for optimization, a metamodel is often proposed. This work examines a set of metamodeling techniques for the design optimization of composite laminates with variable stiffness. Three case studies are considered. The first two pertain to the fiber path design of a plate under uniform compression. The third concerns the optimization of a composite cylinder under pure bending. Four metamodeling methods, namely Polynomial Regression, Radial Basis Functions, Kriging and Support Vector Regression, are tested, and their performance is compared. Accuracy, robustness, and suitability for integration within an optimization framework are the appraisal criteria. The results show that the most accurate and robust models in exploring the design space are Kriging and Radial Basis Functions. The suitability of Kriging is the highest for a low number of design variables, whereas the best choice for a high number of variables is Radial Basis Functions.
\end{abstract}

Keywords: Metamodel, Optimization, Variable stiffness composite, Automated Fiber Placement. 


\section{Introduction}

Automated fiber placement (AFP) is a technology capable of placing fibers along a curvilinear path, thereby resulting in a variable stiffness laminate. The structural benefits of variable stiffness laminates are achieved by tailoring the material properties in directions that are more favourable to carry loads within the laminates. To fully exploit the advantages of a variable stiffness design, it is often appropriate to systematically formulate the design problem within an optimization framework. The objective functions to optimize might be one or more mechanical properties, such as buckling and in-plane stiffness. Since the fiber orientation continuously changes within the laminate of a variable stiffness design, the evaluation of the structural properties via finite element simulation is often very time-consuming $[1,2]$. Furthermore, the optimization process might require thousands of function evaluations to locate a near optimal solution, a requirement that makes the process computationally expensive. To alleviate this problem, one may resort to an approximation concept, also called a metamodel [3, 4]. Significantly cheaper to evaluate, the metamodel is substituted and used in place of a high fidelity finite element simulation. As a result, the metamodel can significantly reduce the time required to run the optimization.

In the literature, there are several successful applications of metamodeling techniques in the optimization of traditional composite laminates with straight fibers. For example, Radial Basis Functions [5], second order polynomials [6], and Neural Networks [7] were shown to be effective in reducing the time to find the maximum buckling load of a composite stiffened panel. Liu et al. [8] used a cubic response surface combined with a two-level optimization technique to maximize the buckling load of a composite wing. Lee and Lin [9, 10] used trigonometric functions as the base functions to build a metamodel for the stacking sequence optimization of a composite propeller. Integrated into a genetic algorithm (GA), the metamodel demonstrated benefits by reducing the number of GA iterations. Kalnins et al. [11] compared the performance of Radial Basis Functions, multivariate adaptive regression splines, and polynomials, to optimize the post-buckling of a damaged composite stiffened structure. They concluded that the methods under investigation have cross-validation error lower than $10 \%$; thus, they can be efficiently integrated into an optimization framework. In another attempt, Lanzi and Giavotto [12] compared the performance of Radial Basis Functions, Neural Networks, and Kriging 
metamodels in a multi-objective optimization problem for maximum post-buckling load and minimum weight of a composite stiffened panel. The methods were found to yield similar results and none of them was identified as being significantly superior.

While there is a considerable amount of existing research on the use of metamodels for constant stiffness composite design, only a few attempts look at their application in variable stiffness design. Among those worthy to mention are the following: the optimization of a variable stiffness laminate in vibration [13], the buckling load of a variable stiffness composite cylinder [14], and the simultaneous optimization of the buckling load and in-plane stiffness of a variable stiffness laminate $[2,15]$. While these works demonstrate the potential of a given metamodel in reducing the computational burden of the optimization process, they are just a firs attempt. No recommendation about metamodel selection for variable stiffness composites exists. Furthermore, metamodel performance is problem dependent and the best metamodel is unknown at the outset [16].

This work presents a comparative study on the application of the most widely used metamodeling methods -polynomial regression, Radial Basis Functions, Kriging, and Support Vector Regression, for the optimization of variable stiffness composite. The goal is to offer insight into the selection of the most appropriate metamodel for the optimization of laminated composites with varying fiber angles. We examine three case studies: the buckling load and inplane stiffness of a variable stiffness composite plate under uniform compression for two layup designs, and a variable stiffness composite cylinder under pure bending. The advantages and disadvantages of the metamodels are then investigated using the following criteria:

- Accuracy: the degree of closeness of a metamodel prediction to that quantity of the true function over the design range of interest. Multiple metrics, namely R-square, relative average absolute error, and relative maximum absolute error are used to assess the metamodels' accuracy.

- Robustness: the capability of a metamodel to persistently achieve high accuracy for dissimilar problems. In this work, the robustness of a metamodel method is measured by evaluating its average accuracy for the entire set of test problems.

- Suitability: the degree of the effectiveness of integrating a metamodel into an evolutionary optimization algorithm. To measure this criterion, the performance of 
metamodel-assisted optimization algorithms in the actual improvement of the solution is compared via a series of numerical experiments on the case studies.

The remainder of this work is organized as follows: the data sampling method and the different size of the sample data to investigate its effect on the metamodel accuracy are explained in Section 2. Section 3 gives a background on metamodel construction techniques and their characteristics. The metrics to evaluate the local and global metamodel accuracy are discussed in Section 4. Test problems for variable stiffness composite that can be manufacturable via AFP are then described in Section 5. Finally, the metamodels under investigation are assessed and recommendations are presented in Section 6.

\section{Data sampling}

Data sampling, referred to as design of experiments (DoE), is the first step in the construction of a metamodel. The selection of the sample points and the size of the sample have a significant effect on the metamodel accuracy.

Sacks et al. [17] stated that sample points for simulated experiments should be chosen to fill the design space rather than to concentrate on the boundaries of the design space. The reason is that computer experiments are deterministic and thus involve systematic errors, whereas physical experiments involve random errors. Following this observation, in this work a Latin Hypercube method is used to generate training data that are space filling. In addition, to average out the dependency of the metamodels accuracy on the sampling method, we use five DoEs to construct each metamodel.

Besides the sampling method, the sample size also has an influence on metamodel accuracy. To investigate the metamodel accuracy with respect to the sample size, small and large sample sizes are examined as suggested by Jin et al [18]. Table 1 shows the sample sizes and the number of confirmation data points used to measure metamodel accuracy with respect to the sample size.

\section{Metamodeling techniques}

As mentioned in the introduction, there is a variety of techniques that can be used to construct a metamodel. This section gives a background on the most common methods: Polynomial 
Regression (PR); Radial Basis Functions (RBF); Kriging (KRG); and Support Vector Regression (SVR).

\subsection{Polynomial Regression (PR)}

A second-order polynomial can be expressed as

$$
\tilde{y}(\mathrm{x})=\beta_{0}+\sum_{i=1}^{n} \beta_{i} x_{i}+\sum_{i=1}^{n} \beta_{i i} x_{i}^{2}+\sum_{i=1}^{n} \sum_{j=i+1}^{n} \beta_{i j} x_{i} x_{j}
$$

Where $\beta_{0}, \beta_{\mathrm{i}}, \beta_{\mathrm{ii}}$ and $\beta_{\mathrm{ij}}(\mathrm{i}, \mathrm{j}=1, \ldots, \mathrm{n})$ are the regression coefficients, $\mathrm{x}_{\mathrm{i}}(\mathrm{i}=1, \ldots, \mathrm{n})$ are the design variables, and $\tilde{y}$ denotes the approximate value for the objective function. The coefficients of the metamodel are evaluated by fitting the model to the training data using the least squares method [19]. The second order PR has a smoothing capability, a feature that ensures fast convergence for noisy functions and thus is suitable for integration in an optimization framework. Yet, this characteristic can bring inaccuracy if there is need to surrogate highly non-linear functions [18]. Obviously, a higher order polynomial can be used to construct a more accurate metamodel; nevertheless, instabilities may arise and also a large number of training data is required to fit such a high order polynomial [20].

\subsection{Radial Basis Functions (RBF)}

The RBF method uses a combination of basis functions expressed in terms of the Euclidean distance between sample data points to construct a metamodel [21]. The RBF model can be written as

$$
\tilde{y}(x)=\sum_{i=1}^{n} w_{i} \psi\left(\left\|x-x_{i}\right\|\right)
$$

where $x_{i}(i=1, \ldots, n)$ are the design variables, $\psi$ is the basis function and $\mathrm{w}_{\mathrm{i}}(\mathrm{i}=1, \ldots, \mathrm{n})$ are the basis function weights evaluated by fitting the model to the training data, \|.\| denotes the Euclidean distance between two sample data points, and $\tilde{y}$ is the approximate value of the objective function [4]. The basis function weights, $w_{i}$, can be calculated by enforcing the interpolation condition in Eq. (2). This results in a linear system of equations 


$$
\mathbf{y}=\psi \mathbf{w}
$$

where $\mathbf{y}$ is the vector of function values at training data, $\mathbf{w}$ is the vector of basis function weights, and $\psi$ is a matrix, also known as Gramian matrix of design variable values defined by

$$
\boldsymbol{\psi}=\left|\begin{array}{cccc}
\psi\left(x_{1}, x_{1}\right) & \psi\left(x_{1}, x_{2}\right) & \cdots & \psi\left(x_{1}, x_{n c}\right) \\
\psi\left(x_{2}, x_{1}\right) & \psi\left(x_{2}, x_{2}\right) & \cdots & \psi\left(x_{2}, x_{n c}\right) \\
\vdots & \vdots & \ddots & \vdots \\
\psi\left(x_{n c}, x_{1}\right) & \psi\left(x_{n c}, x_{2}\right) & \cdots & \psi\left(x_{n c}, x_{n c}\right)
\end{array}\right|
$$

In this study a multiquadratic function, $\psi(r)=\sqrt{r^{2}+s^{2}}$, where $r=\left\|x-x_{i}\right\|$ and $s$ is the RBF width parameter, are considered as the basis function. When the design variables are scaled to the range $[0,1]$, the $\mathrm{RBF}$ parameter can be selected independently from the values of the design variable.

\subsection{Kriging (KRG)}

The Kriging method uses a combination of a trend function $\mathrm{P}(\mathrm{x})$, which is usually a polynomial (e.g. linear or quadratic), and a departure from the trend function, $\mathrm{Z}(\mathrm{x})$, to construct a metamodel.

$$
\tilde{y}(x)=\sum_{i=1}^{n} \beta_{i} P_{i}(x)+Z(x)
$$

The $\mathrm{Z}(\mathrm{x})$ is assumed to be "a realization of a stochastic process with a mean of zero and a correlation function given by" [17]

$$
\operatorname{cor}\left[Z\left(x_{i}\right), Z\left(x_{j}\right)\right]=\sigma^{2} R\left(x_{i}, x_{j}\right)
$$

where $\sigma^{2}$ is the process variation and $R\left(x_{i}, x_{j}\right)$ is the correlation, which usually takes the form of a Gaussian radial basis function as [17]

$$
R\left(x_{i}, x_{j}\right)=\exp \left(-\sum_{i=1}^{n} \theta_{i}\left|x_{i}-x_{j}\right|^{p_{i}}\right)
$$


It should be noted that in Eq. (7) the correlation parameters of the basis functions, i.e. $\theta_{i}$ and $p_{i}$, were identical for all dimensions in the RBF model, whereas they could be different for each dimension in a Kriging model. Although these additional parameters make KRG more flexible than RBF, they should be obtained by maximizing a likelihood function [4]. The major disadvantage of Kriging is the need to solve the maximization problem, which makes the KRG computationally expensive if the number of design variables is high.

\subsection{Support Vector Regression (SVR)}

SVR is a special version of the Support Vector Machine (SVM) developed for regression analysis. SVR uses a subset of data samples, support vectors, to construct a metamodel that has a maximum deviation of $\varepsilon$ from the function value of each training data [22]. For a linear regression, the SVR model can be written as

$$
\tilde{y}(x)=\langle w \cdot x\rangle+b
$$

where $\tilde{y}$ is the approximate value of the objective function at $x, w$ represents a vector of weights, $b$ is the bias term, and $\langle$.$\rangle denotes the inner product. Instead of minimizing the empirical risk on$ the training data during the fitting process, SVR minimizes an upper bound on the expected risk using an $\varepsilon$-insensitive loss function, as proposed by [22]

$$
L(x)= \begin{cases}0 & \text { if }|y(x)-\tilde{y}(x)| \leq \varepsilon \\ |y(x)-\tilde{y}(x)|-\varepsilon & \text { otherwise }\end{cases}
$$

SVR performs a linear regression $\varepsilon$-insensitive loss function, at the same time, tries to reduce the model complexity by minimizing the norm of the weighting vector, $\|w\|^{2}$.

$$
\begin{aligned}
& \min \frac{1}{2}\|w\|^{2} \\
& \text { s.t. }\left\{\begin{array}{l}
y_{i}-\left\langle\mathbf{w} \cdot \mathbf{x}_{\mathbf{i}}\right\rangle-b \leq \varepsilon \\
\left\langle\mathbf{w} \cdot \mathbf{x}_{\mathbf{i}}\right\rangle+b-y_{i} \leq \varepsilon
\end{array}\right.
\end{aligned}
$$

It should be noted that there might not be a function that satisfies the condition in equation (10). Thus, slack variables are incorporated into the optimization problem as 


$$
\begin{aligned}
& \min \frac{1}{2}\|w\|^{2}+C \sum_{i=1}^{n}\left(\xi_{i}+\xi_{i}^{*}\right) \\
& \text { s.t. }\left\{\begin{array}{l}
y_{i}-\left\langle\mathbf{w} \cdot \mathbf{x}_{\mathbf{i}}\right\rangle-b \leq \varepsilon+\xi_{i}^{*} \\
\left\langle\mathbf{w} \cdot \mathbf{x}_{\mathbf{i}}\right\rangle+b-y_{i} \leq \varepsilon+\xi_{i} \\
\xi_{i}, \xi_{i}^{*} \geq 0
\end{array}\right.
\end{aligned}
$$

The regularization parameter, $C$, determines the trade-off between the model complexity and the degree for which deviation larger than $\varepsilon$ is tolerated in Eq. (10). A nonlinear regression can be achieved by replacing the $\langle$.$\rangle in Eq. (8) with a kernel function, K$, [22] as

$$
\tilde{f}(x)=\sum_{i=1}^{n}\left(\alpha_{i}-\alpha_{i}^{*}\right) K\left(\mathbf{x}_{\mathbf{i}}, \mathbf{x}\right)+b
$$

In the case studies examined in this paper, a Gaussian kernel function is used and $\varepsilon$ and $C$ parameters are chosen based on the recommendation proposed by Cherkassky and Ma [23]. For more details on SVR, the interested reader may refer to [22-24].

\section{Accuracy Metrics}

There is a variety of metrics to measure the accuracy of a metamodel. Cross-validation error is a popular choice. It relies on training data and does not require additional sample data to calculate the error. Yet, cross-validation error was found to potentially lead to a biased estimate of the error [25, 26]. In addition, Lin [27] stated that "cross validation is an insufficient measurement for metamodel accuracy". Hence, in this study, we opt for other accuracy metrics which require

additional sample data (Table 1). These include R-square, relative average absolute error (RAAE), and relative maximum absolute error (RMAE) [18, 27].

(a) R-square:

$$
R^{2}=1-\frac{\sum_{i=1}^{n}\left(y_{i}-\hat{y}_{i}\right)^{2}}{\sum_{i=1}^{n}\left(y_{i}-\bar{y}_{i}\right)^{2}}
$$


$\hat{y}_{i}$ denotes the value predicted by the metamodel, $y_{i}$ represents the true value, and $\bar{y}_{i}$ is the mean of the true values at confirmation points. A larger R-square denotes higher accuracy of the metamodel.

(b) Relative average absolute error (RAAE)

$$
R A A E=\frac{\sum_{i=1}^{n}\left|y_{i}-\hat{y}_{i}\right|}{n \times S T D}
$$

where STD stands for standard deviation. This metric is a good indicator of the global accuracy of a metamodel. The closer to zero RAAE is, the more accurate the metamodel.

(c) Relative maximum absolute error (RMAE)

$$
R M A E=\frac{\max \left(\left|y_{1}-\bar{y}_{1}\right|,\left|y_{1}-\bar{y}_{1}\right|, \ldots,\left|y_{n}-\bar{y}_{n}\right|\right)}{S T D}
$$

In this case, accuracy increases with decreasing values of RMAE.

$\mathrm{R}^{2}$ and RAAE indicate the overall accuracy of a metamodel over the entire design space. A high RMAE value indicates large error in a region of the design space.

\section{Test problems of laminate composite}

This section examines three problems, each involving the design of variable stiffness composite parts, which can be manufactured by AFP. The first two deal with the design and optimization of a composite plate, with prescribed layup configurations; the third is about the optimum design of a composite cylinder.

\subsection{Composite plates with curvilinear fibers}

A variable stiffness composite plate can be designed by a curvilinear fiber path that varies linearly along the $x$-axis of the plate (Figure 1) and can be formulated as 


$$
\theta(x)=\frac{2\left(T_{1}-T_{0}\right)}{a}|x|+T_{0}
$$

where $\theta$ represents the fiber orientation, $a$ denotes the plate width, $T_{0}$ and $T_{1}$ are the fiber angles at the plate center $(x=0)$ and the plate edges $(x= \pm a / 2)$, respectively [28]. A single layer with this fiber path definition can be specified with two design variables, i.e., $T_{0}$ and $T_{1}$, where $T_{0}=T_{1}$ represents a straight fiber case [29].

Case 1. A $0.254 \times 0.406 \mathrm{~m}(10 \times 16 \mathrm{in})$ rectangular plate with a 16 -ply balanced symmetric laminate subjected to a uniform end shortening along the y-direction is designed by using the fiber path definition given in (13). Concerning the boundary conditions, the transverse edges are considered free (Figure 1b) for in-plane displacement and all edges are simply supported against out-of-plane movement. The in-plane stiffness and the buckling load of a plate with a $[ \pm \theta(x)]_{4 s}$ layup are considered as the objective functions. This test problem has two design variables, i.e. $T_{0}$ and $T_{1}$, that allow for visual comparison of the metamodel accuracy.

Case 2. The second case study is a similar plate with a $\left[ \pm \theta_{1}(x) / \pm \theta_{2}(x) / \pm \theta_{3}(x) / \pm \theta_{4}(x)\right]_{S}$ layup, with 8 design variables, i.e. $\mathrm{T}_{0 \mathrm{i}}(\mathrm{i}=1, \ldots, 4)$ and $\mathrm{T}_{1 \mathrm{i}}(\mathrm{i}=1, \ldots, 4)$. This problem is considered to investigate the effect of the size of the problem on metamodels performance. Similar to the first test problem, the buckling load and equivalent in-plane stiffness of the variable stiffness plates are considered as the objective functions. The plates are designed with the following material properties: $E_{x}=181 \mathrm{MPa}, E_{y}=10.27 \mathrm{MPa}, G_{x y}=7.17 \mathrm{MPa}$, and $v_{x y}=0.28$.

\subsection{A cylinder with a curvilinear fiber path}

Case 3 is a composite cylinder with a diameter of $0.6096 \mathrm{~m} \mathrm{(24}$ in) and a length of $0.8122 \mathrm{~m} \mathrm{(32}$ in) [14] considered to investigate the performance of metamodels for a problem with 3-D geometry (Figure 2).

A variable stiffness cylinder is designed by specifying multiple segments for the linear fiber angle variation. The fiber angle is varied as a function of the circumferential coordinate over multiple segments of the cylinder [14]. 


$$
\theta(\varphi)=T_{i}+\frac{T_{i+1}-T_{i}}{45} \times[\varphi-(i-1) \times 45] \quad i \in[1,2,3, \ldots, 8]
$$

In Eq. (17), $\theta$ represents fiber orientation, which depends only on the circumferential angle $(\varphi)$ and is independent from the longitudinal and radial direction. $T_{i}$ is the fiber orientation in each segment as it is indicated in Figure 2. The layup is a 16-ply symmetric and balanced laminate $[ \pm \theta(\varphi)]_{4 s}$ with 8 design variables, $\mathrm{T}_{\mathrm{i}}(\mathrm{i}=1, \ldots, 8)$, and material properties as the ones given in Section 5.1. The cylinder is assumed to be under pure bending with tension at the top and compression at the bottom surfaces. The buckling load and overall stiffness of the cylinder are considered as the objective functions.

\section{Assessment of metamodels}

This section presents a comparative study of metamodels in predicting the buckling load and inplane stiffness of the test problems described in section 5. As mentioned, the performance of the metamodels depends on the number of training data. Thus for each case, we generated 5 sets of DoEs with small and large size; the metamodels were entirely refitted to each of them. In other words, we built a total of 120 metamodels by using the metamodeling techniques described in section 3, and different sample data. The metrics, Eqs (13-15), were then used to calculate the accuracy of each metamodel.

As previously mentioned in section 5.1, the first case study is a variable stiffness plate with $[ \pm \theta(x)]_{4 s}$ layup. This test problem has only two design variables, i.e. $T_{0}$ and $T_{1}$ that allow to qualitatively compare the metamodel accuracy via a contour plot. Figure 3 shows the buckling load of the variable stiffness plate of Case 1. Figure 3a is the plot of the true model, Figures $3 b-e$ illustrate the iso-buckling regions obtained with the metamodels. As can be seen, KRG and RBF provide a good accuracy, as opposed to SVR and PR, which cannot capture the real behavior.

Multiple bar charts can be used to quantitatively compare the effect of the sample size on metamodel accuracy. Figure 4 shows bars representing the average of a given metric (R2, RAAE, and RMAE) for the metamodels under investigation. We gather that the accuracy of all the metamodels generally improves with a large set of training data. For both small and large sets 
of sample data, accuracies of KRG and RBF are very similar and better than both PR and SVR. Figure $4 \mathrm{c}$ shows that the size of the sample data has a higher impact on the RMAE of PR compared to the other methods. We can observe that PR produces the least accurate model even for a large set of training data.

Another metric to assess a metamodel performance is robustness. Robustness refers to the capability of a metamodel to be accurate in a range of problems. Figure 5 shows box plots of accuracy metrics for all metamodels. A box plot is defined by a lower quartile (25\%), median (50\%), and upper quartile (75\%) values. The extended lines represent the minimum and maximum of values. The height of a box (the space between lower and upper quartile) represents the robustness of the method. The smaller the box size, the higher the robustness. By comparing the box sizes, we found that KRG is more robust than RBF, whereas their median is very close. PR and SVR are the least robust methods. Therefore from these plots we gather that KRG and RBF are preferable methods to construct metamodels for variable stiffness composites.

\subsection{Metamodel-assisted optimization}

The results from the previous section help us to gain insight into the accuracy of the metamodels. A large number of sample data is generally required to produce an accurate metamodel of variables stiffness laminates that can span the entire design space. It should be noted that a metamodel with good global accuracy is not necessarily able to capture the optimum values of the true function. It is thus important to select a metamodel that is accurate in regions where optimum solutions most likely can be found. The performance of a metamodel in other regions of the design space are thus of minor concern for metamodel-assisted optimization [30].

In this section, the suitability of metamodels (the degree of the effectiveness of integrating a metamodel into an evolutionary optimization algorithm) is illustrated using a series of numerical experiments. In these experiments, a metamodel is integrated into a genetic algorithm, where the metamodel is updated at each generation of the optimization process as suggested in [30]. The suitability of each metamodel is then illustrated by comparing the best obtained solution at each generation.

The first case study requires the optimization of a variable stiffness laminate with a $[ \pm \theta(x)]_{4 s}$ layup (2 variables only). The buckling load is a unimodal function (Figure 3) and the exact 
location of the optimum is known. Figure 6 shows the distance between the optimum of the metamodel and the optimum of the true function during the optimization process, averaged over five trials for each case. It can be seen that KRG predicts the true optimum after the first generation of the GA, whereas RBF reaches that optimum after the second generation. PR and SVR reach a point very close to the true optimum but even after several updates they cannot predict the exact optimum. The results show that all metamodels improve the performance of the GA by approaching the neighbourhood of the optimum at an early stage of the GA generations. However, only KRG and RBF can locate the exact optimum within an early number of generations. Moreover, it can be seen in Figure 6 that after 6 generations, there is no benefit of adding more points to the training data and updating the metamodels. It is worth noting that in terms of metrics, as it was shown in Figure 4, PR is less accurate than SVR; however, it has a better performance when integrated into the GA. Therefore, a poor metamodel in terms of accuracy metrics does not necessarily have a poor performance when integrated into an optimization algorithm.

For the remaining case studies, the optimum design is not known. Thus to compare the performance of the metamodel-assisted optimization algorithms, we use the following relative improvement

$$
\mathrm{I}=\frac{y_{I B S}-y_{M A O}}{y_{I B S}}
$$

where $y_{I B S}$ is the initial best solution and $y_{M A O}$ is the optimum solution found at each generation by the metamodel-assisted optimization algorithm, and $\mathrm{I}$ is the actual improvement over the initial best solution. The actual improvement shows how well a metamodel-assisted optimization performs. When there is no improvement in the solution $\mathrm{I}=0$; ; on the other hand, $\mathrm{I}>0$ represents an improvement in the solution. A larger I means a higher improvement in the solution [31]. The results of the optimization averaged over five trials for each case are shown in Figure 7.

Similar to the previous case, all metamodels have the effect of enhancing the performance of the optimization process by improving the accuracy of the solution at low number of generations. For example, the final best solution found by GA is obtained by all metamodel-assisted GA after 3 generations only. In other words, for the case studies shown here, the use of the metamodels 
decreases of one third the number of generations required by the genetic algorithm. The difference between metamodel-assisted algorithms is significant at early generations and decreases during the optimization process. This might be explained by examining the evolution of the accuracy during the generations; all models can locate the neighbourhood of the optimum solution after 5-6 generations. Hence, further iteration cannot significantly increase their accuracy. In general, RBF followed by KRG outperforms other methods. In contrast to the previous case, early in the optimization process SVR performs better than PR, yet its superiority over PR diminishes as the optimization proceeds.

\section{Conclusions}

This work has compared the performance of alternative metamodeling methods using multiple criteria for the design optimization of variable stiffness composite laminates. The metamodels performance has been assessed in three case studies. Their accuracy and robustness in constructing an approximation of the buckling load and in-plane stiffness have been measured. The suitability of each metamodel for integration into an optimization framework has also been studied. KRG and RBF had the highest accuracy. In terms of robustness, both KRG and RBF provided the best results, where KRG has been slightly better than RBF. In terms of suitability, KRG has shown the best performance for problems with low number of design variables, whereas RBF has been the most appropriate method for a high number of variables. It is found that the use of an appropriate metamodel in a metamodel-assisted genetic algorithm decreases the number of iterations to one-third compared to a genetic algorithm.

In this study, the size of the sample data has been considered fixed. Further investigation is needed to determine the minimum number of sample data required to reach a certain level of accuracy for a given metamodel. In addition, further work is needed to investigate the role of kernel and basis functions respectively for KRG and RBF metamodels.

\section{References}

[1] Blom A, Setoodeh S, Hol J, Gürdal Z. Design of variable-stiffness conical shells for maximum fundamental eigenfrequency. Computers \& Structures. 2008;86:870-8.

[2] Arian Nik M, Fayazbakhsh K, Pasini D, Lessard L. Surrogate-based multi-objective optimization of a composite laminate with curvilinear fibers. Composite Structures. 2012;94:2306-13.

[3] Wang GG, Shan S. Review of metamodeling techniques in support of engineering design optimization. Journal of Mechanical Design. 2007;129:370-80. 
[4] Forrester A, Keane A. Recent advances in surrogate-based optimization. Progress in Aerospace Sciences. 2009;45:50-79.

[5] Irisarri FX, Laurin F, Leroy FH, Maire JF. Computational strategy for multiobjective optimization of composite stiffened panels. Composite Structures. 2011;93:1158-67.

[6] Rikards R, Abramovich H, Kalnins K, Auzins J. Surrogate modeling in design optimization of stiffened composite shells. Composite Structures. 2006;73:244-51.

[7] Bisagni C, Lanzi L. Post-buckling optimisation of composite stiffened panels using neural networks. Composite Structures. 2002;58:237-47.

[8] Liu B, Haftka RT, Akgün MA. Two-level composite wing structural optimization using response surfaces. Structural and Multidisciplinary Optimization. 2000;20:87-96.

[9] Lee Y-J, Lin C-C. Regression of the response surface of laminated composite structures. Composite Structures. 2003;62:91-105.

[10] Lin C-C, Lee Y-J. Stacking sequence optimization of laminated composite structures using genetic algorithm with local improvement. Composite Structures. 2004;63:339-45.

[11] Kalnins K, Rikards R, Auzins J, Bisagni C, Abramovich H, Degenhardt R. Metamodeling methodology for postbuckling simulation of damaged composite stiffened structures with physical validation. International Journal of Structural Stability \& Dynamics. 2010;10:705-16.

[12] Lanzi L, Giavotto V. Post-buckling optimization of composite stiffened panels: Computations and experiments. Composite Structures. 2006;73:208-20.

[13] Vandervelde T, Milani AS. Layout optimization of a multi-zoned, multi-layered composite wing under free vibration. Proceedings of SPIE, the International Society for Optical Engineering San Diego, CA, USA; 2009.

[14] Blom AW, Stickler PB, Gürdal Z. Optimization of a composite cylinder under bending by tailoring stiffness properties in circumferential direction. Composites Part B: Engineering. 2010;41:157-65.

[15] Fayazbakhsh K, Arian Nik M, Pasini D, Lessard L. Defect layer method to capture effect of gaps and overlaps in variable stiffness laminates made by Automated Fiber Placement. Composite Structures. 2013;97:245-51.

[16] Viana FAC, Gogu C, Haftka RT. Making the most out of surrogate models: tricks of the trade. ASME Conference Proceedings. 2010;2010:587-98.

[17] Sacks J, Welch WJ, Mitchell TJ, Wynn HP. Design and analysis of computer experiments. Statistical science. 1989;4:409-23.

[18] Jin R, Chen W, Simpson TW. Comparative studies of metamodelling techniques under multiple modelling criteria. Structural and Multidisciplinary Optimization. 2001;23:1-13.

[19] Myers RH, Montgomery DC. Response surface methodology: process and product optimization using designed experiments: J. Wiley, 2002.

[20] Barton RR. Metamodels for simulation input-output relations. Proceedings of the 24th conference on Winter simulation. Arlington, Virginia, USA;: ACM; 1992. p. 289-99.

[21] Dyn N, Levin D, Rippa S. Numerical Procedures for Surface Fitting of Scattered Data by Radial Functions. SIAM Journal on Scientific and Statistical Computing. 1986;7:639-59.

[22] Vapnik VN. Statistical learning theory: Wiley, 1998.

[23] Cherkassky V, Ma Y. Practical selection of SVM parameters and noise estimation for SVM regression. Neural networks. 2004;17:113-26.

[24] Gunn SR. Support Vector Machines for Classification and Regression. Technical Report, Image Speech and Intelligent Systems Research Group, University of Southampton; 1997.

[25] Picard RR, Cook RD. Cross-validation of regression models. Journal of the American Statistical Association. 1984:575-83.

[26] Varma S, Simon R. Bias in error estimation when using cross-validation for model selection. BMC bioinformatics. 2006;7:91.

[27] Lin Y. An efficient robust concept exploration method and sequential exploratory experimental design. Atlanta, GA: Georgia Institute of Technology, 2004. 
[28] Gürdal Z, Olmedo R. In-plane response of laminates with spatially varying fiber orientations: variable stiffness concept. AIAA journal. 1993;31:751-8.

[29] Gürdal Z, Tatting BF, Wu CK. Variable stiffness composite panels: effects of stiffness variation on the in-plane and buckling response. Composites Part A: Applied Science and Manufacturing. 2008;39:911-22.

[30] Ong YS, Nair PB, Keane AJ. Evolutionary optimization of computationally expensive problems via surrogate modeling. AIAA journal. 2003;41:687-96.

[31] Felipe V, Raphael H. Surrogate-based Optimization with Parallel Simulations using the Probability of Improvement. 13th AIAA/ISSMO Multidisciplinary Analysis Optimization Conference. Fort Worth, Texas, USA: AIAA; 2010. 
Table 1: Experimental design for test problems (adapted from [18]).

\begin{tabular}{cc}
\hline Sample size & Number of training data points \\
\hline Small set & $10 n(9$ if $n=2)$ \\
Large set & $\frac{3(n+1)(n+2)}{2}$ \\
\hline Confirmation data points & 300 for test problem 1,1000 for test problems 2 and 3 \\
\hline
\end{tabular}




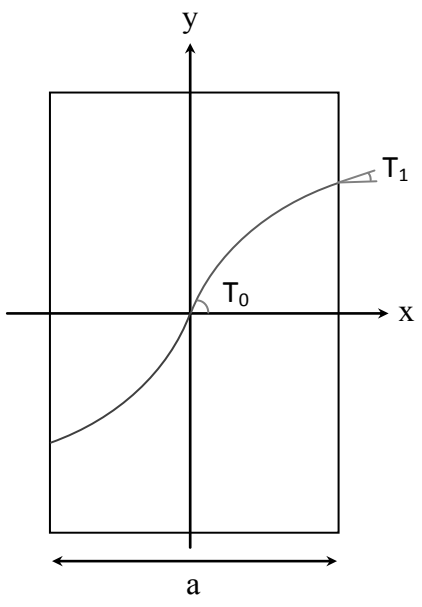

(a)

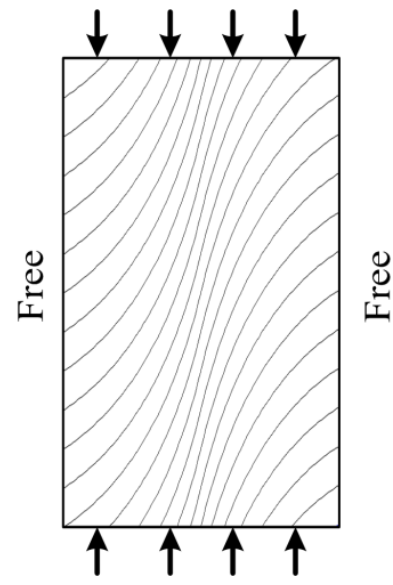

(b)

Figure 1: (a) A curvilinear fiber path that varies linearly along the $x$-axis; (b) Test case load and boundary conditions. 


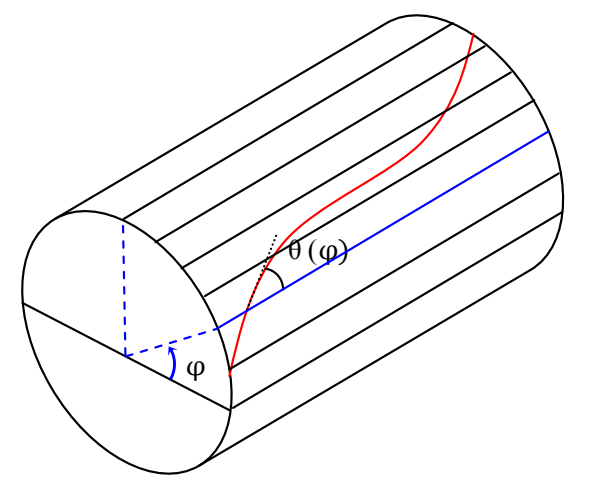

(a)

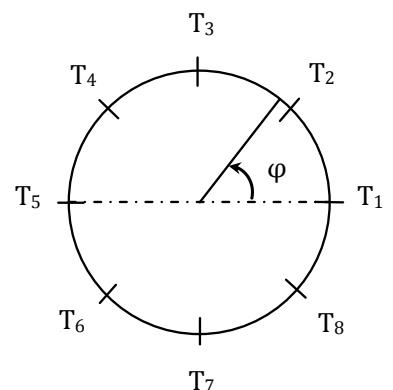

(b)

Figure 2. (a) Fiber angle definition for a variable stiffness cylinder. (b) Design variables per ply (reproduced from $[14])$. 


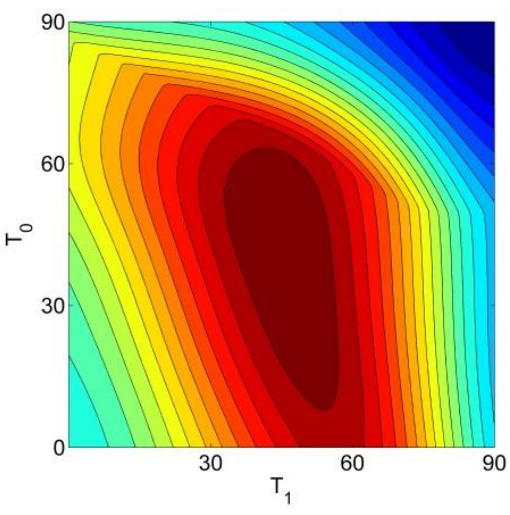

(a)

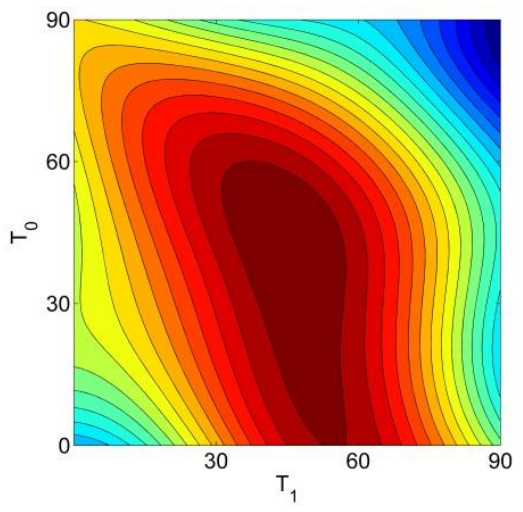

(b)

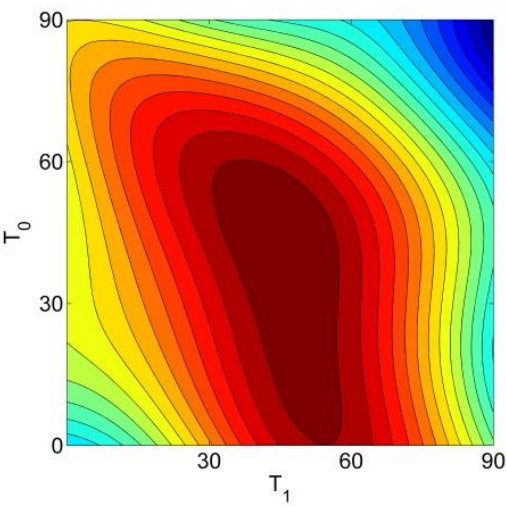

(c)

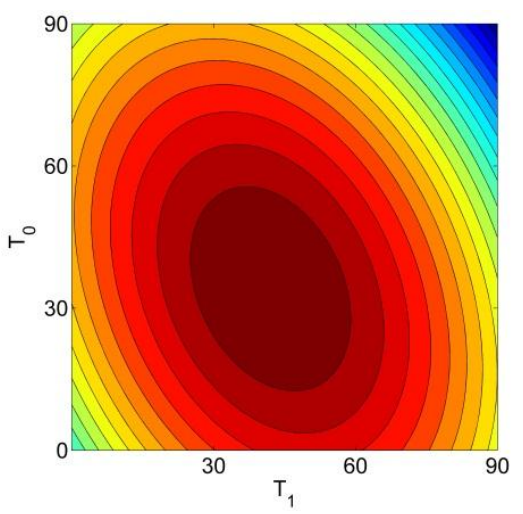

(d)

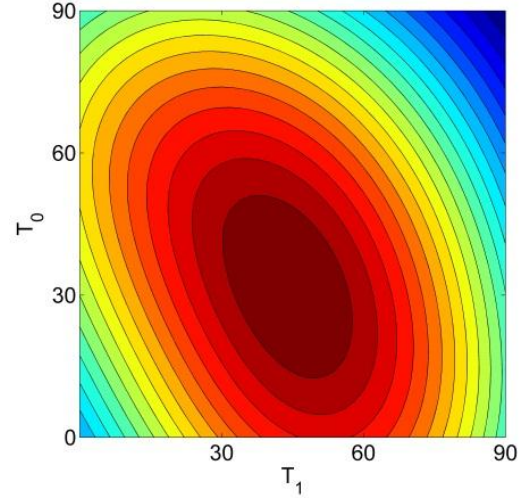

(e)

Figure 3. Contour plots for the buckling load of a variable stiffness composite plate with 2 design variables. (a) True model; (b) KRG; (c) RBF; (d) PR; (e) SVR. 


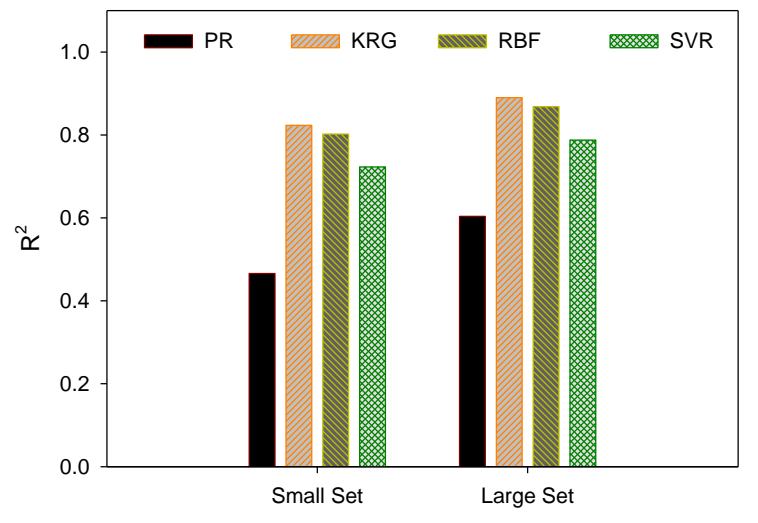

(a)

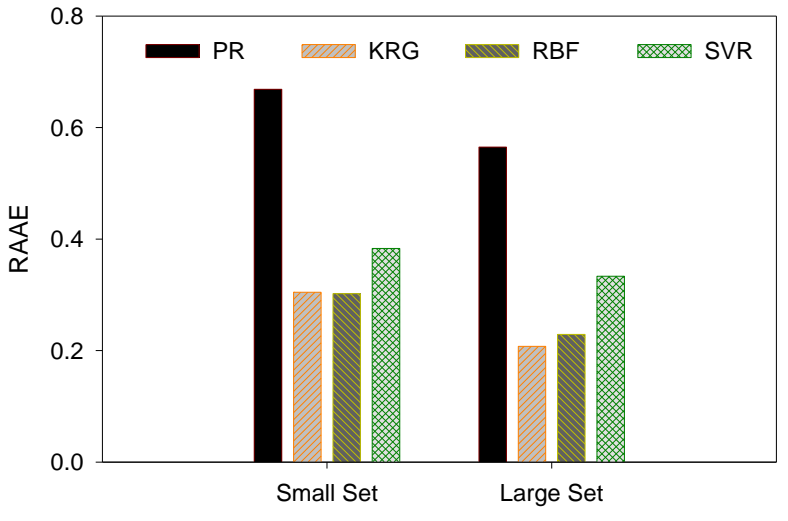

(b)

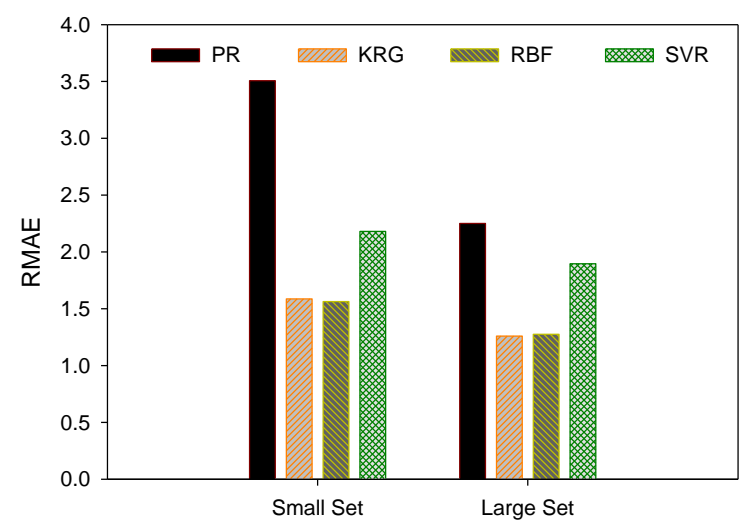

(c)

Figure 4. The effect of sample size on accuracy metrics for PR, KRG, RBF, and SVR metamodels: (a) R-square; (b) RAAE; (c) RMAE. 


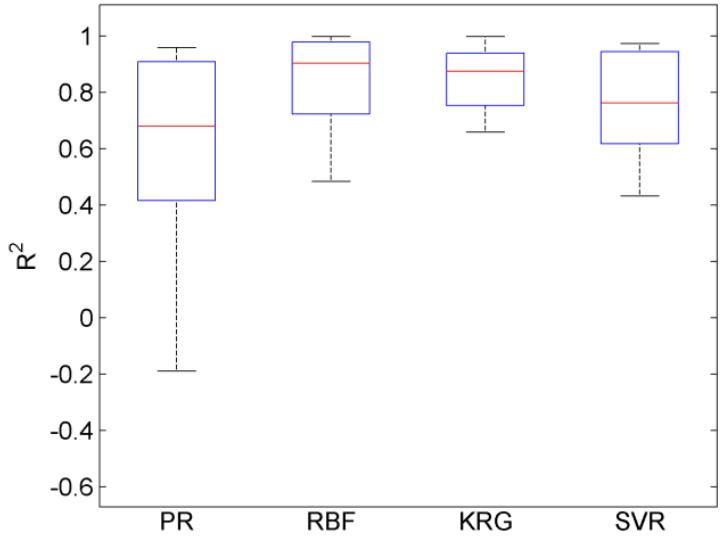

(a)

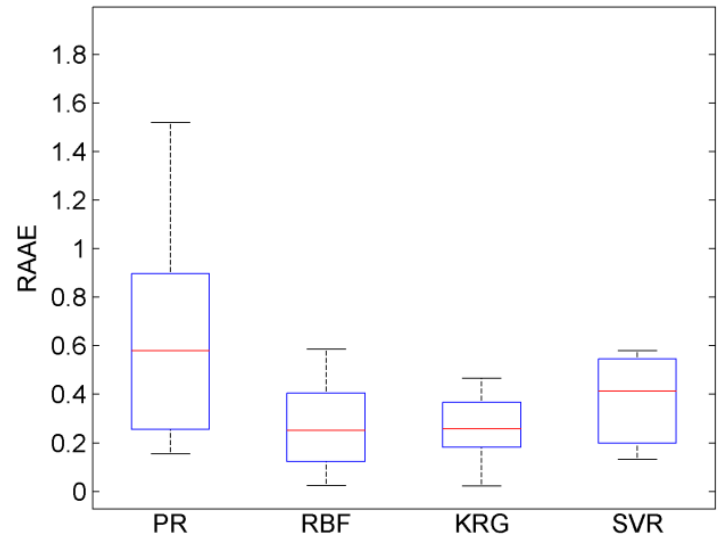

(b)

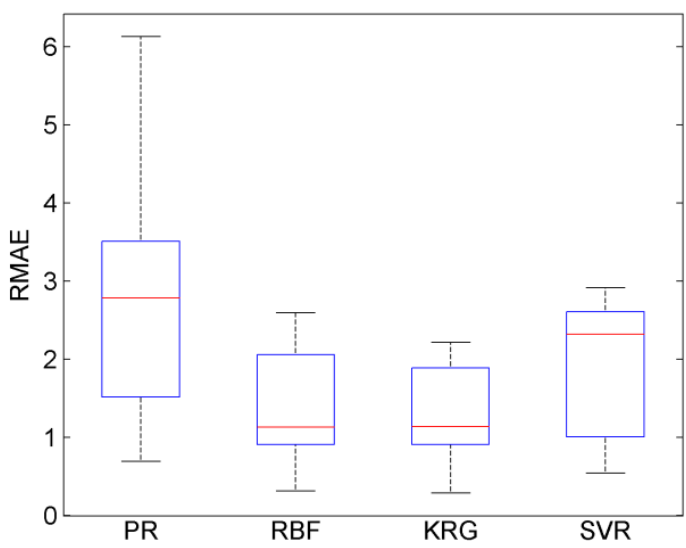

(c)

Figure 5. Performance metrics of each metamodel constructed for the variable stiffness composite test problems examined in section 5. (a) R-square; (b) RAAE; (c) RMAE. 


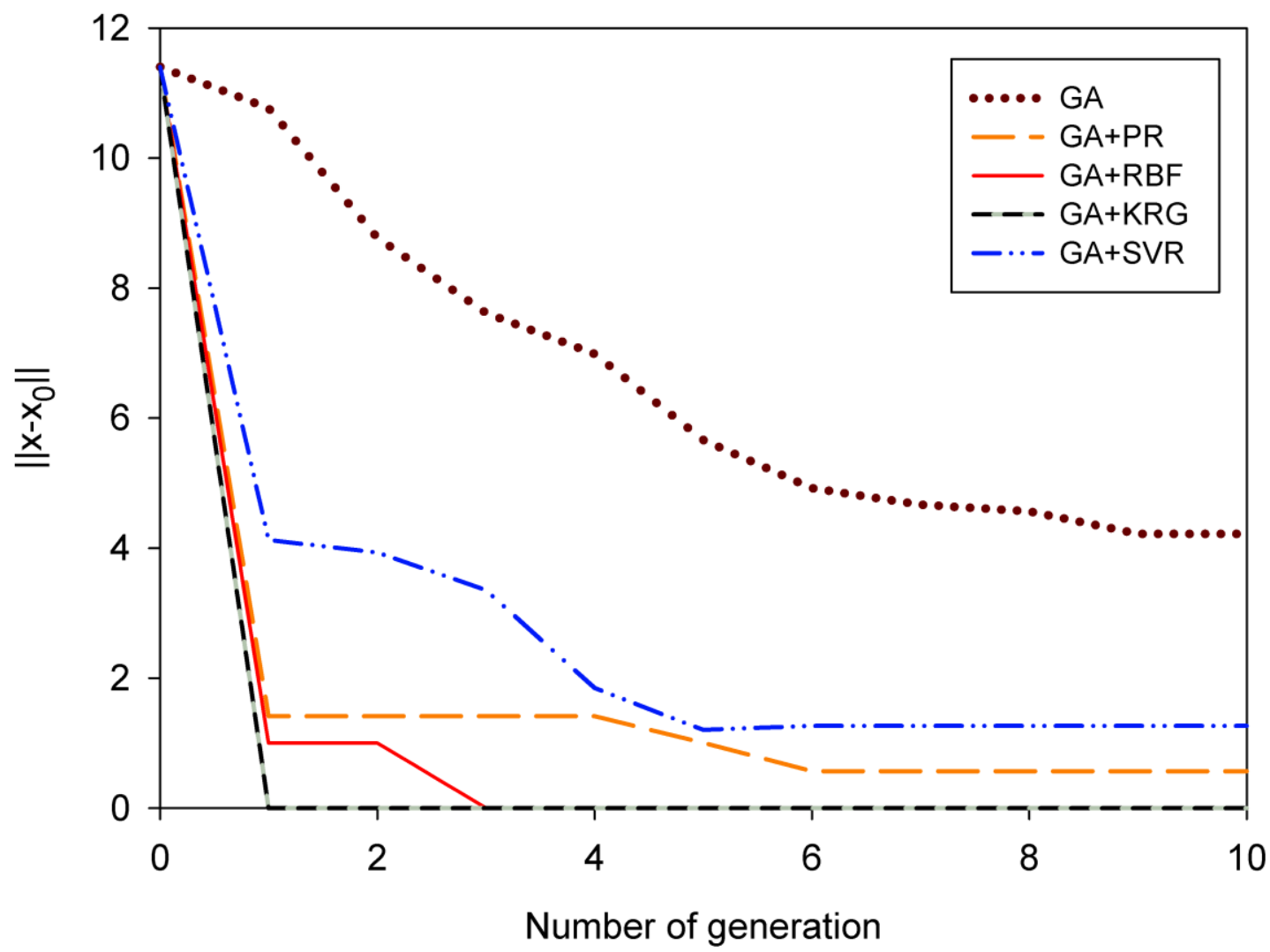

Figure 6. Metamodel performance as a function of the number of iterations. Maximization of the buckling load for a $[ \pm \theta(x)]_{4 s}$ composite laminate. 


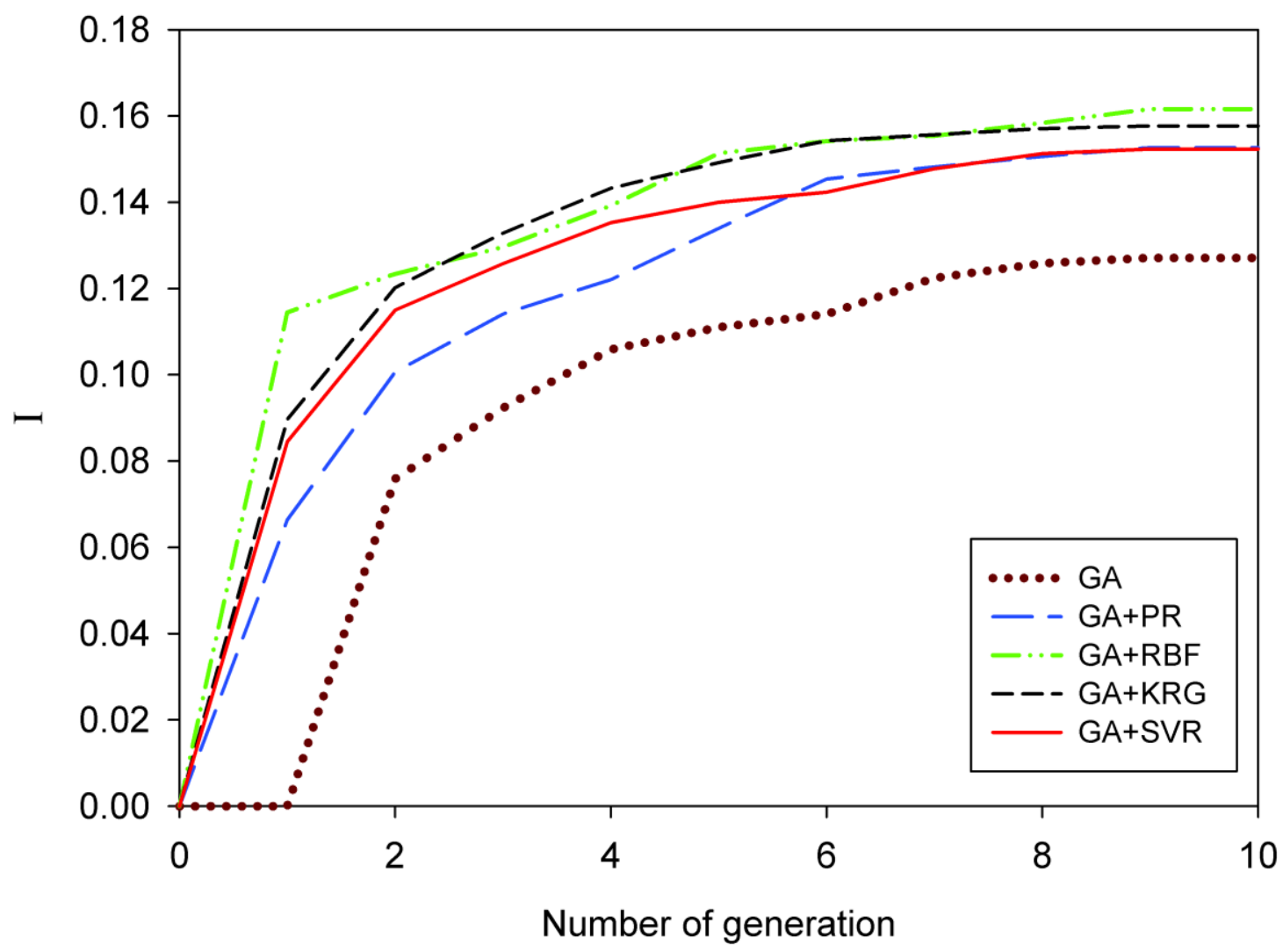

Figure 7. Performance of the metamodels- for the optimization of variable stiffness laminates. 